\title{
Chronic disseminated candidiasis manifesting as hepatosplenic abscesses among patients with hematological malignancies
}

Chien-Yuan Chen ${ }^{1 *}$, Aristine Cheng ${ }^{2}$, Feng-Ming Tien ${ }^{1,3,4}$, Po-Chu Lee ${ }^{4,5,6}$, Hwei-Fang Tien ${ }^{1,7}$, Wang-Huei Sheng ${ }^{2,7^{*}}$ and Yee-Chun Chen ${ }^{2,7}$

\begin{abstract}
Background: The outcomes of deep-seated abscesses attributed to chronic disseminated candidiasis (CDC) in patients with hematological malignancies have rarely been reported in recent years.

Methods: We retrospectively reviewed and analyzed the data of patients with hematological malignancies who received a diagnosis of CDC at a medical center in Taiwan between 2008 and 2013.

Results: Sixty-one patients (32 men and 29 women) were diagnosed with CDC. The median age was 51 years (range: 18-83). The overall incidence of CDC was 1.53 per 100 patient-years in patients with hematological malignancies between 2008 and 2013. The highest incidence of CDC was 4.3 per 100 patient-years for acute lymphoblastic leukemia, followed by 3.6 for acute myeloid leukemia. We detected 3 (4.9\%) proven, 13 (21.3\%) probable, and 45 (73.8\%) possible cases of CDC. A total of 13 patients had positive blood cultures for Candida species: C. tropicalis (8), C. albicans (2), C. glabrata (2), and C. famata (1).

The median duration of antifungal treatment was 96 days (range: 7-796 days). Serial imaging studies revealed that the resolution rate of CDC was $30.0 \%$ at 3 months and $54.3 \%$ at 6 months. Five patients (8.2\%) had residual lesions that persisted beyond one year. A multivariate analysis of the 90-day outcome revealed that shock was the only independent prognostic factor of 90-day survival in patients with CDC.

Conclusion: The incidence of CDC did not decrease between 2008 and 2013. Patients with acute leukemia had a higher risk of CDC than those with other hematological malignancies. Imaging studies conducted at 6 months after diagnosis revealed that only half of the patients showed complete resolution. CDC requires prolonged treatment, and serial imaging at 6 months interval is suggested. Shock is the only independent prognostic factor of 90-day survival in patients with CDC.
\end{abstract}

Keywords: Chronic disseminated candidiasis (CDC), Hepatosplenic candidiasis, Hematological malignancy, Resolution of image, Survival

\footnotetext{
* Correspondence: chienyuanchen@ntu.edu.tw; whsheng@ntu.edu.tw

${ }^{1}$ Division of Hematology, Department of Internal Medicine, National Taiwan

University Hospital, No. 7 Chung-Shan South Road, Taipei 10002, Taiwan

${ }^{2}$ Division of Infectious Disease, Department of Internal Medicine, National

Taiwan University Hospital, Taipei, Taiwan

Full list of author information is available at the end of the article
}

(c) The Author(s). 2019 Open Access This article is distributed under the terms of the Creative Commons Attribution 4.0 International License (http://creativecommons.org/licenses/by/4.0/), which permits unrestricted use, distribution, and reproduction in any medium, provided you give appropriate credit to the original author(s) and the source, provide a link to the Creative Commons license, and indicate if changes were made. The Creative Commons Public Domain Dedication waiver (http://creativecommons.org/publicdomain/zero/1.0/) applies to the data made available in this article, unless otherwise stated. 


\section{Background}

Candida is a common pathogen worldwide and in patients with cancer, high morbidity and mortality are attributed to invasive candidiasis [1, 2]. Chronic disseminated candidiasis (CDC) is a unique clinical manifestation of invasive candidiasis; it usually develops during recovery from neutropenia after chemotherapy and affects organs such as the liver and spleen in patients with acute leukemia [3-6]. The exact pathogenesis of CDC is unknown. Studies have proposed that Candida species colonizing the bowel, invade and seed to hepatosplenic sinusoids from the portal splenic bloodstream following chemotherapy-induced mucosal damage $[7,8]$. CDC is defined as small, peripheral, targetlike abscesses (bull's-eye lesions) in the liver or spleen demonstrated on computed tomography $(\mathrm{CT})$, magnetic resonance, or ultrasound imaging; it is accompanied by an elevated level of serum alkaline phosphatase. Supporting microbiological characteristics are not required to fulfill the European Organization for Research and Treatment of Cancer (EORTC) criteria for CDC $[9,10]$.

The reported incidence of $\mathrm{CDC}$ has ranged from 2.0 to $7.4 \%$ in patients with acute leukemia $[5,6,11-15]$. Rammaert et al. proposed that the incidence has reduced in the last decade, which was possibly due to the prophylactic and preemptive use of antifungal agents [4]. However, in recent years, few studies have analyzed the epidemiology of CDC in patients with hematological malignancies. Although patients with hematological malignancies other than acute leukemia who developed CDC have been observed in our clinical practice and in a previous study [6], the epidemiology of CDC in these patients has rarely been described.

The treatment duration of CDC depends on clinical symptoms and signs, and the resolution of abscesses from imaging studies. The Infectious Diseases Society of America (IDSA) recommends that therapy should be continued until lesions have resolved on repeat imaging, which is usually after several months [16]. The premature discontinuation of antifungal therapy may lead to a relapse of CDC [16]. However, studies conducting serial imaging follow-up of CDC are limited. Thus, in this study, we retrospectively reviewed and analyzed the clinical manifestation, treatment, serial imaging follow-up, and prognostic factors of CDC in 2083 patients with hematological malignancies between 2008 and 2013.

\section{Methods}

The National Taiwan University Hospital (NTUH) is a 2800-bed teaching hospital in the metropolitan area of Taipei that provides both primary and tertiary care. The medical records of patients admitted to the hematological wards of the NTUH from January 1, 2008, to December 31, 2013, were retrospectively reviewed.
The inclusion criteria were patients with acute myeloid leukemia (AML), acute lymphoblastic leukemia (ALL), lymphoma, multiple myeloma (MM), chronic myeloid leukemia $(\mathrm{CML})$, myeloproliferative neoplasm (MPN), chronic lymphocytic leukemia (CLL), myelodysplastic syndrome (MDS), or severe aplastic anemia (SAA). Patients with other hematological diseases such as hemolytic anemia, idiopathic thrombocytopenia, hemophilia, or coagulation disorders were excluded. The methods have already been described in our previous studies [17].

Imaging studies using CT and ultrasonography and other examinations were performed if clinically indicated. This retrospective study was approved by the Research Ethics Committee of NTUH (Institutional Review Board No: 20160613132RIND), and informed consent was waived for this analysis based on approval by the Research Ethic Committee, as the data was analyzed anonymously.

\section{Diagnosis of chronic disseminated candidiasis}

CDC was diagnosed according to the EORTC and the Mycosis Study Group consensus criteria to define invasive fungal infection in this study, and CDC cases were categorized as proven, probable, or possible $[9,10]$.

\section{Antifungal prophylaxis and antifungal treatment}

At NTUH, no antifungal prophylaxis agent was administered to patients with hematological malignancies, except for a nystatin oral suspension, between 2008 and 2013. Patients awaiting allogeneic stem cell transplantation received micafungin for prophylaxis before neutrophil recovery on the transplantation ward. Antifungal treatments were provided according to the local guidelines of the Infectious Diseases Society of Taiwan $[18,19]$.

\section{Statistical analysis}

We used the Chi-square test for categorical comparisons of the data. Significant predictors in the univariate analysis were included in a forward, stepwise multiple logistic-regression model to identify the most crucial risk factors for 90-day mortality. A Cox proportional hazard analysis was used to determine the relative contribution of various factors to the risk of 90-day mortality. In the multivariate analysis, factors with $P$ values $<0.2$ in the univariate analysis were selected for the Cox proportional hazard regression analysis. A survival analysis was performed using the Kaplan-Meier estimator and a logrank test. A $P$ value of $<0.05$ indicated statistical significance. All statistical analyses were performed using SPSS for Windows (Version 18.0, SPSS Inc., Chicago, Il, USA). 


\section{Results}

Clinical characteristics of 2083 hematological malignancy patients with and without CDC (hepatosplenic microabscesses)

A total of 2083 patients with hematological malignancies between 2008 and 2013 were included in this study, and 61 of these patients were diagnosed with CDC based on clinical and imaging criteria. In total, 968 patients were women, and the median age was 56 years (range: 15-97). A significant proportion of patients $(n=607)$ were aged 65 years-old or more. These elderly patients ( $\geq 65$ years) had a lower risk of CDC than younger patients $(1.5 \%$ vs $3.5 \%, P=0.010)$. In this cohort, 397 patients underwent allogeneic stem cell transplantation, among whom 26 patients developed CDC (4 before transplantation and 22 after transplantation). Overall, patients who underwent stem cell transplantation had a higher risk of CDC than those who did not undergo transplantation $(6.5 \%$ vs $2.1 \%, P<0.001)$. The other clinical characteristics of the patients are listed in Table 1 . The frequency of underlying diabetes mellitus, hepatitis $\mathrm{B}$, and liver cirrhosis was not significantly different among patients with and without CDC.

The incidence of $\mathrm{CDC}$ in patients with hematological malignancies was 1.53 per 100 patient-years between 2008 and 2013 (Fig. 1). The incidence of CDC was highest at 4.3 per 100 patient-years (PYs) in patients with ALL, followed by 3.6 per 100 PYs in patients with AML, 0.5 per $100 \mathrm{PYs}$ in patients with CML or MPN, 0.4 per
100 PYs in patients with lymphoma, 0.4 per 100 PYs in patients with MDS or SAA, 0.2 per 100 PYs in patients with myeloma. No cases of CDC were detected among patients with CLL.

\section{Clinical manifestation in 61 patients with chronic disseminated candidiasis}

In total, 61 patients (32 men and 29 women) were diagnosed with CDC. The median age was 51 years (range: 18-83). The underlying hematological malignancies were AML $(n=38)$, ALL $(n=13)$, lymphoma $(n=7)$, CML/MPN $(\mathrm{n}=1), \operatorname{MDS} / \mathrm{SAA}(\mathrm{n}=1)$, and myeloma $(n=1)$. Four patients underwent allogeneic stem cell transplantation before the diagnosis of CDC, and 22 patients underwent allogeneic stem cell transplantation after the diagnosis of the CDC. CDC was detected in all 61 patients either by computed tomography or by abdominal ultrasonography. By imaging studies, CDC was detected in 56 (94.9\%) of 59 patients through computed tomography and in 44 (75.9\%) of 58 patients through abdominal ultrasonography. Thirty-nine patients had positive findings on both $\mathrm{CT}$ and abdominal ultrasound imaging. The most common organs involved were the liver (58/61, 95.1\%), spleen (34/61, 55.7\%), and incidentally, the kidney $(14 / 61,23 \%)$. Twenty-seven patients underwent liver biopsy, and only 3 patients yielded a confirmatory result $(11.1 \%)$; candidal hyphae were detected in the pathological specimens of 2 patients and $C$. albicans was recovered by tissue culture in 1 patient. A

Table 1 Clinical characteristics of 2083 patients with and without chronic disseminated candidiasis (CDC)

\begin{tabular}{|c|c|c|c|}
\hline & $\begin{array}{l}\text { Patients with CDC } \\
(n=61, \%)\end{array}$ & $\begin{array}{l}\text { Patients without CDC } \\
(n=2022, \%)\end{array}$ & $p$ \\
\hline Age & & & 0.010 \\
\hline Age $>=65$ & $9(1.5)$ & $598(98.5)$ & \\
\hline Age $<65$ & $52(3.5)$ & $1424(96.5)$ & \\
\hline Gender & & & 0.897 \\
\hline Men & $32(2.9)$ & $1083(97.1)$ & \\
\hline Women & $29(3.0)$ & $939(97.0)$ & \\
\hline Allogeneic transplantation & & & $<0.001$ \\
\hline Yes & $26(6.5)$ & $371(93.5)$ & \\
\hline No & $35(2.1)$ & $1651(97.9)$ & \\
\hline Diabetes Mellitus & & & 0.849 \\
\hline Yes & $7(2.5)$ & $276(97.5)$ & \\
\hline No & $54(3.0)$ & $1746(97.0)$ & \\
\hline Hepatitis B carrier & & & 1.000 \\
\hline Yes & $9(3.0)$ & $296(97.0)$ & \\
\hline No & $52(2.9)$ & $1726(97.0)$ & \\
\hline Liver Cirrhosis & & & 1.000 \\
\hline Yes & $0(0.0)$ & $8(100.0)$ & \\
\hline No & $61(2.9)$ & $2014(97.1)$ & \\
\hline
\end{tabular}




\section{Incidence}

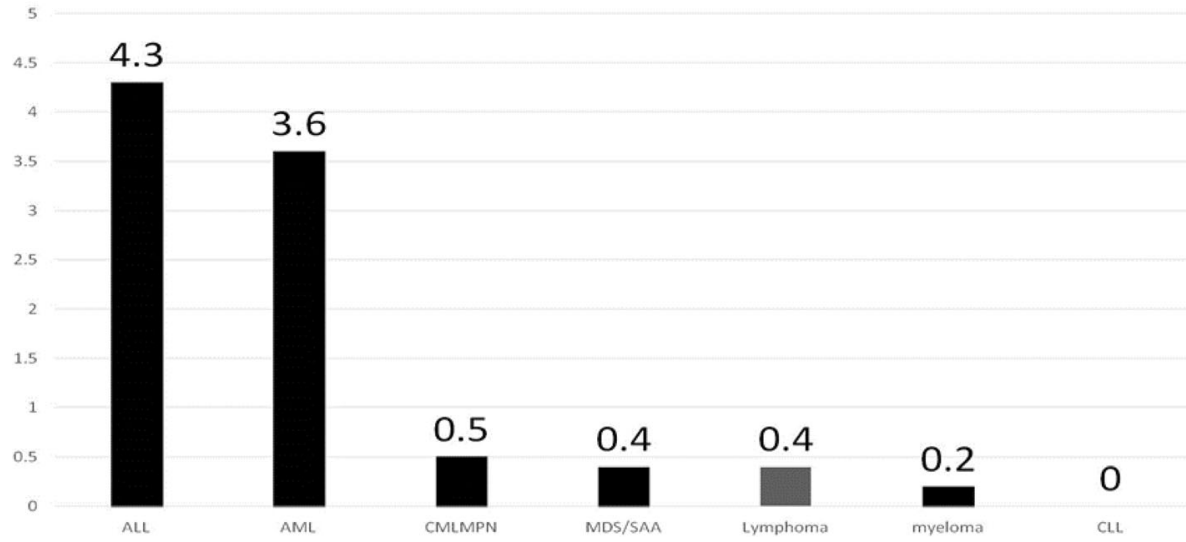

Fig. 1 Incidence of hematological malignancies in patients with chronic disseminated candidiasis

total of 13 patients had concomitant positive blood cultures yielding C. tropicalis (8 patients), C. albicans (2 patients), C. glabrata (2 patients), and C. famata (1 patient), respectively. In this study, we detected 3 (4.9\%) proven, $13(21.3 \%)$ probable, and $45(73.8 \%)$ possible cases of CDC.

\section{Antifungal treatment and resolution of chronic disseminated (hepatosplenic) candidiasis by imaging} A variety of antifungal agents were administered to patients with CDC (Table 2). Thirty-five (57.3\%) patients received fluconazole and 9 (14.8\%) received amphotericin $\mathrm{B}$ as their initial antifungal agent. The median duration of antifungal treatment was 96 days (range: 7-796 days). The median interval between serial imaging studies was 187 days (range: 1-1274). Each patient received a median number of 3.5 CT follow-up studies (range: 012 ) and a median number of 3.3 ultrasound follow-up studies (range: $0-8$ ). All patients underwent at least 3 imaging studies, except for 2 patients due to premature deaths: 1 patient died 11 days after the diagnosis of $\mathrm{CDC}$, and the other patient had persistent lesions at the second CT scan and died 40 days after the diagnosis of CDC. At 3-months following the diagnosis of CDC, 12 of 40 patients $(30.0 \%)$ had resolution of their hepatosplenic or renal abscesses on imaging, and 28 of 40 patients (70.0\%) had residual abscesses. At 6-months, 19 of 35 patients (54.3\%) showed complete resolution of abscesses on imaging, and 16 of 35 patients $(45.7 \%)$ had residual abscesses. Overall, 35 of 61 patients $(57.3 \%)$ had documented resolution of CDC in this study. The median time to resolution of microabscesses was 161 days (range: 16-1274 days). Five of 61 patients (8.2\%) had persistent residual $\mathrm{CDC}$ on imaging studies beyond one year. Time to documented resolution of CDC by imaging was analyzed using the Kaplan-Meier method
(Table 2). Neutropenia at the diagnosis of CDC $(P=$ $0.005)$ was associated with a more rapid resolution of CDC in imaging studies. Sixteen (84.2\%) of 19 patients recovered from neutropenia, but $3(15.8 \%)$ patients died within 90 days.

\section{Mortality at 90 days in 61 patients with chronic disseminated candidiasis}

We analyzed the 90-day mortality of 61 patients with CDC (Table 3). A total of 12 patients (19.7\%) died within 90 days. A univariate analysis using the Kaplan-Meier method revealed that both the type of hematological disease $(P<0.001)$ and initial antifungal agents $(P<0.001)$, and timing of allogeneic transplantation $(P<0.001)$ influenced 90-day survival. Age, gender, underlying diabetes, hepatitis $\mathrm{B}$, neutropenia, and thrombocytopenia were not associated with 90-day survival. A multivariate analysis with a Cox hazard regression revealed that shock was the only independent prognostic factor of 90day survival in patients with $C D C(P=0.016$, odds ratio $<0.001,95 \% \mathrm{CI}<0.001-0.184)$.

\section{Discussion}

In this study, 51 (6.3\%) of 808 patients with acute leukemia were diagnosed with CDC. This finding is consistent with that of our previous study, which revealed that 37 of $500(7.4 \%)$ patients with acute leukemia developed CDC. Despite recent advances in the use of preemptive or prophylactic antifungals, the incidence of CDC has not decreased compared with earlier studies (incidence: $2.0-7.4 \%$ ) $[5,6,11-15]$. The incidence of CDC in patients with hematological malignancies varied depending on the subtype; it was higher among patients with ALL and AML but was lower among those with CML/MPN, lymphoma, MDS/SAA, or multiple myeloma, and no CDC was recorded in patients with CLL. 
Table 2 Univariate analysis of factors of the resolution of CDC in imaging studies at 6 months

\begin{tabular}{lll}
\hline Factor & $\begin{array}{l}\text { Residual lesions } \\
(n=26)\end{array}$ & $\begin{array}{l}\text { Reso } \\
(n=18\end{array}$ \\
\hline Gender & & 18 \\
Man & 14 & 17 \\
Woman & 12 & 28 \\
Age & & 7 \\
$\quad<65$ years & 24 & \\
$\geqq 65$ years & 2 &
\end{tabular}

Acute leukemia Resolution $(n=35)$

Yes

No

Hypertension

No

Diabetes mellitus

Yes

Hepatitis B carrier

Hepatitis C

Yes

No

Proven CDC

Yes

No

Involved more than 2 organs

Yes

No

High dose chemotherapy

Yes

No

Preceding HSCT

Yes

No

Remission status

Yes

No

Neutropenia at diagnosis of $\mathrm{CDC}$

Yes

No

Thrombocytopenia at diagnosis of CDC

Yes

No

Adequate initial antifungal
30

5

0.550

29

2

33

0.080

5

30

0.069

19

16

0.459

15

20

2

33

15

20

0.005

14

21

0.750

29 
Table 2 Univariate analysis of factors of the resolution of CDC in imaging studies at 6 months (Continued)

\begin{tabular}{cll}
\hline Factor & $\begin{array}{l}\text { Residual lesions } \\
(n=26)\end{array}$ & $\begin{array}{l}\text { Resolution } \\
(n=35)\end{array}$ \\
\hline Yes & 25 & 34 \\
No & 1 & 1 \\
\hline
\end{tabular}

Most importantly, CDC was not limited to patients with acute leukemia. Other clinical characteristics such as younger age, and allogeneic transplantation were associated with a higher risk of developing hepatosplenic microabscesses.

The resolution of abscesses in imaging studies is crucial for optimizing patient care. IDSA guidelines state that therapy should continue until the lesions are resolved on repeat imaging, which is usually after several months. The premature discontinuation of antifungal therapy can lead to relapses [16]. However, studies on abscess resolution in serial imaging studies are limited. Berlow et al. reported that 2 patients showed abscess resolution in CT imaging studies after 5 and 9 months [20]. Kauffman et al. reported abscess resolution in imaging studies from 4 weeks to approximately 4-5 months, and 1 patient showed a persistent abscess for 13 months [21]. Görg et al. reported that of 6 patients with CDC, 5 had resolved lesions at 2-6 months, and 1 had a persistent lesion for more than 36 months, as demonstrated by ultrasound images [22]. In this study, 12 of 40 patients $(30.0 \%)$ showed abscess resolution in imaging studies at 3 months, and 19 of 35 patients (54.3\%) showed abscess resolution at 6 months. Five of 61 patients $(8.2 \%)$ had persistent residual abscesses for more than 1 year. Half of patients had lesions after more than 6 months. Therefore, we suggest that imaging should be performed for most patients with CDC at 3-6 monthly intervals with at least six months of follow-up to guide therapy duration, with prolonged follow-up duration (beyond one year for certain patients with residual lesions at 6 months).

No consensus has been reached on the required duration of antifungal treatment. Parenteral antifungal agents are usually necessary at the time of diagnosis and are substituted with oral agents thereafter (fluconazole, itraconazole, voriconazole, and posaconazole). In this study, the median duration of antifungal treatment was 96 days (range: 7-796 days). As the antifungal agent administered was heterogeneous and the study retrospective in nature, the comparative therapeutic effect was difficult to determine. However, half of the abscesses on imaging studies resolved at 6 months. A treatment course of 3-6 months is warranted if lesions persist in serial imaging studies. When the factors associated with the resolution of $\mathrm{CDC}$ were analyzed, only recovery of neutropenia was correlated with the rapid resolution of
CDC in imaging studies. Nineteen patients had neutropenia at the time of CDC diagnosis; patients whose neutropenia subsequently recovered had significantly faster imaging documented resolution than patients whose neutrophil counts did not recover $(p<0.001)$. This result implicates recovery of neutropenia is associated with rapid resolution of the microabscesses. By contrast, the hepatosplenic or renal abscesses of patients without neutropenia at the time of CDC diagnosis recovered less rapidly since the underlying immune defects predisposing to invasive fungal infections may be less easily reversed than by recovery of absolute neutrophil counts. Shoham et al. proposed that the propensity for the persistence of the fungus in infected tissues may be a consequence of cell-mediated immune dysregulation with the suppression of Th1 and the overexpression of Th2 responses [23]. The mechanisms underlying the clearance of $\mathrm{CDC}$ requires further investigation.

Diagnostic accuracy is a major problem in CDC. CDC without documented candidemia often remains unrecognized [24]. The mannan antigen and antimannan antibody have been studied as biomarkers for diagnosing CDC in patients with ALL [25]. Evidencebased reviews of currently available nonculture-based diagnostic markers of invasive candidiasis in patients with hemato-oncological malignancies are scant, and studies on the use of mannan antigen and anti-mannan antibody tests are limited to reports by the European Conference on Infections in Leukemia (ECIL) [26]. For mannan antigen and anti-mannan antibody tests, the optimal cutoffs for positivity, as well as the number of samples required for confirmation of positivity, requires validation [27]. Although the EORTC reached a consensus on the diagnosis of $\operatorname{CDC}[9,10]$, the low rate of proven cases by tissue pathology or culture, low blood culture yield rates, prohibitive radiation effects of $\mathrm{CT}$, severely impacts diagnostic accuracy. A recent study showed that small splenic abscesses on fluorodeoxyglucose positron emission tomography (FDG-PET) images could be missed on corresponding CT images [27]. Whether FDG-PET improves diagnostic accuracy over $\mathrm{CT}$ and MR imaging also requires further clarification.

This study has several limitations that are also present in clinical practice. This was a retrospective study that analyzed cases of CDC between 2008 and 2013, and the initial antifungal agents administered were heterogeneous. In this study, fluconazole was the was most 
Table 3 Kaplan-Meier analysis of prognostic factors of 90-day survival in 61 patients with chronic disseminated candidiasis

\begin{tabular}{llll}
\hline Age & $\begin{array}{l}\text { Total } \\
(n=61)\end{array}$ & $\begin{array}{l}\text { Alive } \\
(n=49)\end{array}$ & $\begin{array}{l}\text { D } \\
(n=\end{array}$ \\
Age $>=65$ years & & & 1 \\
Age $<65$ years & 9 & 8 & 5 \\
Gender & 52 & 41 & 7 \\
Men & & & 4 \\
Women & 32 & 27 & 5 \\
Hematological subtype & 29 & 22 & 2 \\
AML & & & 0 \\
ALL & 38 & 34 & 0 \\
Lymphoma & 13 & 5 & 0 \\
CML/MPN & 7 & 1 & 1 \\
CLL & 1 & 0 & 1 \\
Myeloma & 0 & 0 & \\
MDS/SAA & 1 & 1 &
\end{tabular}

Preceding allogeneic transplantation

p
$\mathrm{p}$

0.539

11

Yes

5

No

56

1

48

0.650

Neutropenia $<=500 / \mathrm{mm}^{3}$

Thrombocytopenia

Platelet $>=20000 / \mathrm{mm}^{3}$

Proven or Probable CDC

$\begin{array}{ll}\text { Yes } & 16 \\ \text { No } & 45\end{array}$

Initial anti-fungal agent

Amphotericin B

Fluconazole

Itraconazole

Voriconazole

Posaconazole

Caspofungin

Micafungin

Anidulafungin

none

\section{2}

0.492

37

Hepatitis B carrier

Yes

No

Diabetes mellitus

7

0 1

1

1

\section{0}

\section{3}

1

2

10 
Table 3 Kaplan-Meier analysis of prognostic factors of 90-day survival in 61 patients with chronic disseminated candidiasis (Continued)

\begin{tabular}{clllll}
\hline & $\begin{array}{l}\text { Total } \\
(n=61)\end{array}$ & $\begin{array}{l}\text { Alive } \\
(n=49)\end{array}$ & $\begin{array}{l}\text { Death } \\
(n=12)\end{array}$ & $\begin{array}{l}\text { Univariate } \\
p\end{array}$ & $\begin{array}{l}\text { Multivariate } \\
p\end{array}$ \\
\hline Yes & 8 & 0 & 8 & \\
No & 53 & 49 & 4 & \\
\hline
\end{tabular}

Abbreviations: AML acute myeloid leukemia, ALL acute lymphoblastic leukemia, CML chronic myeloid leukemia, MPN myeloid proliferative neoplasm, CLL chronic lymphoid leukemia, MDS myelodysplastic syndrome, SAA severe aplastic anemia, $C D C$ chronic disseminated candidiasis

commonly used first-line antifungal (57.3\%), followed by amphotericin B (14.8\%). The multivariate analysis revealed no significant outcome differences between the first-line antifungal agents. In this study, antifungal treatment was administered according to local guidelines in Taiwan $[18,19]$. Several international guidelines have been developed for invasive candidiasis and antifungal prophylaxis and treatment in patients with hematological malignancies [16, 28, 29]. Accordingly, the Taiwan National Health Insurance provided reimbursement since July 2015 for posaconazole used in the context of antifungal prophylaxis among patients with AML in Taiwan. The incidence of CDC after this secular change is an ongoing study.

The univariate analysis revealed that hematological subtypes, preceding allogeneic transplantation, and administration of initial antifungal agents were poor prognostic factors of CDC. The multivariate analysis indicated that shock was an independent prognostic factor of CDC. In total, 22 patients underwent allogeneic transplantation after the infection was controlled. CDC was not an absolute contraindication to stem cell transplantation $[14,30]$ since confirmation of invasive candidiasis represents a major diagnostic and therapeutic challenge for physicians [31]. Early intervention strategies based on clinical prediction rules and nonculturebased diagnostic assays may improve the outcomes of these healthcare-associated complications.

\section{Conclusion}

The incidence of chronic disseminated (hepatosplenic/ renal) candidiasis did not decrease between 2008 and 2013. Patients with acute leukemia have a higher but not exclusive risk of CDC than those with other hematological malignancies. Repeated imaging studies at six months revealed that the hepatosplenic microabscesses had resolved in only half of the patients and it was necessary to continue therapy beyond six months for the remaining half. According to our findings, CDC requires prolonged treatment and follow-up. Among all criteria, shock is the only independent prognostic factor of 90-day survival in patients with CDC.

\section{Abbreviations}

ALL: Acute lymphoblastic leukemia; AML: Acute myeloid leukemia;

CDC: Chronic disseminated candidiasis; CLL: Chronic lymphocytic leukemia;
CML: Chronic myeloid leukemia; CT: Computed tomography; ECIL: European Conference on Infections in Leukemia; EORTC: European Organization for Research and Treatment of Cancer; FDG-PET: Fluorodeoxyglucose positron emission tomography; IDSA: Infectious Diseases Society of America; MDS: Myelodysplastic syndrome; MM: Multiple myeloma;

MPN: Myeloproliferative neoplasm, SAA: severe aplastic anemia

\section{Acknowledgements}

We thank the staff of the Eighth Core Lab., Department of Medical Research, National Taiwan University Hospital, for their technical support during the study. We thank this manuscript was edited by Wallace Academic Editing.

\section{Authors' contributions}

CYC, WHS, and AC designed, conducted the study and wrote manuscript. YCC, PCL, FMT, HFT recruited the patients and provided the patients' care. All authors read and approved the final manuscript.

\section{Funding}

Not applicable.

\section{Availability of data and materials}

The datasets generated and analyzed during the current study are not publicly available without application to the Research Ethics Committee of National Taiwan University Hospital.

\section{Ethics approval and consent to participate}

This retrospective study was approved by the Research Ethics Committee of NTUH (Institutional Review Board No: 20160613132RIND), and informed consent was waived for this analysis based on approval by the Research Ethic Committee, as the data were analyzed anonymously.

\section{Consent for publication}

Not applicable.

Competing interests

The authors declare that they have no competing interests.

\section{Author details}

'Division of Hematology, Department of Internal Medicine, National Taiwan University Hospital, No. 7 Chung-Shan South Road, Taipei 10002, Taiwan. ${ }^{2}$ Division of Infectious Disease, Department of Internal Medicine, National Taiwan University Hospital, Taipei, Taiwan. ${ }^{3}$ Tai-Cheng Stem Cell Center, National Taiwan University Hospital, Taipei, Taiwan. ${ }^{4}$ Graduate Institute of Clinical Medicine, National Taiwan University, Taipei, Taiwan. ${ }^{5}$ Department of Surgery, National Taiwan University Hospital, Taipei, Taiwan. ${ }^{6}$ Department of Trauma, National Taiwan University Hospital, Taipei, Taiwan. ${ }^{7}$ Department of Medicine, National Taiwan University, College of Medicine, No. 7 Chung-Shan South Road, Taipei 10002, Taiwan.

Received: 8 March 2018 Accepted: 4 July 2019

Published online: 17 July 2019

\section{References}

1. Uzun O, Anaissie EJ. Predictors of outcome in cancer patients with candidemia. Ann Oncol. 2000;11:1517-21.

2. Kullberg BJ, Arendrup MC. Invasive Candidiasis. N Engl J Med. 2015;373: 1445-56. 
3. Kontoyiannis DP, Luna MA, Samuels BI, Bodey GP. Hepatosplenic candidiasis. A manifestation of chronic disseminated candidiasis. Infect Dis Clin N Am. 2000;14:721-39.

4. Rammaert B, Desjardins A, Lortholary O. New insights into hepatosplenic candidosis, a manifestation of chronic disseminated candidosis. Mycoses. 2012;55:e74-84.

5. Sallah S, Wan JY, Nguyen NP, Vos P, Sigounas G. Analysis of factors related to the occurrence of chronic disseminated candidiasis in patients with acute leukemia in a non-bone marrow transplant setting: a follow-up study. Cancer. 2001;92:1349-53.

6. Pagano L, Mele L, Fianchi L, Melillo L, Martino B, D'Antonio D, et al. Chronic disseminated candidiasis in patients with hematologic malignancies. Clinical features and outcome of 29 episodes. Haematologica. 2002;87:535-41.

7. Bow EJ, Loewen R, Cheang MS, Schacter B. Invasive fungal disease in adults undergoing remission-induction therapy for acute myeloid leukemia: the pathogenetic role of the antileukemic regimen. Clin Infect Dis. 1995;21:361-9.

8. van de Veerdonk FL, Kullberg BJ, Netea MG. Pathogenesis of invasive candidiasis. Curr Opin Crit Care. 2010;16:453-9.

9. Ascioglu S, Rex JH, de Pauw B, Bennett JE, Bille J, Crokaert F, et al. Defining opportunistic invasive fungal infections in immunocompromised patients with cancer and hematopoietic stem cell transplants: an international consensus. Clin Infect Dis. 2002;34:7-14.

10. de Pauw B, Walsh TJ, Donnelly JP, Stevens DA, Edwards JE, Calandra T, et al. Revised definitions of invasive fungal disease from the European Organization for Research and Treatment of Cancer/invasive fungal infections cooperative group and the National Institute of Allergy and Infectious Diseases mycoses study group (EORTC/MSG) consensus group. Clin Infect Dis. 2008:46:1813-21.

11. Maksymiuk AW, Thongprasert S, Hopfer R, Luna M, Fainstein V, Bodey GP. Systemic candidiasis in cancer patients. Am J Med. 1984;77(4D):20-7.

12. Blade J, Lopez-Guillermo A, Rozman C, Grañena A, Bruguera M, Bordas J, et al. Chronic systemic candidiasis in acute leukemia. Ann Hematol. 1992;64:240-4.

13. Anttila VJ, Elonen E, Nordling S, Sivonen A, Ruutu T, Ruutu P. Hepatosplenic candidiasis in patients with acute leukemia: incidence and prognostic implications. Clin Infect Dis. 1997;24:375-80.

14. Chen CY, Chen YC, Tang JL, Yao M, Huang SY, Tsai W, et al. Hepatosplenic fungal infection in patients with acute leukemia in Taiwan: incidence, treatment, and prognosis. Ann Hematol. 2003:82:93-7.

15. Karthaus $M$, Hebart $H$, Einsele $H$, Schaefer $H$, Scheel-Walter $H$, Buchheidt $D$, et al. Long-term survival in patients with acute leukemia and chronic disseminated candidiasis despite minimal antileukemic therapy. Haematologica. 2006;91:1422-3.

16. Pappas PG, Kauffman CA, Andes DR, Clancy CJ, Marr KA, Ostrosky-Zeichner L, et al. Clinical practice guideline for the Management of Candidiasis: 2016 update by the Infectious Diseases Society of America. Clin Infect Dis. 2016:62:e1-50.

17. Chen CY, Sheng WH, Tien FM, Lee PC, Huang SY, Tang JL, et al. Clinical characteristics and treatment outcomes of pulmonary invasive fungal infection among adult patients with hematological malignancy in a medical centre in Taiwan, 2008-2013. J Micobiol Immunol Infect. 2018. https://doi. org/10.1016/j.jmii.2018.01.002

18. Infectious Diseases Society of Taiwan; Medical Foundation in Memory of Dr. Deh-Lin Cheng; Foundation of Professor Wei-Chuan Hsieh for Infectious Diseases Research and Education; CY Lee's Research Foundation for Pediatric Infectious Diseases and Vaccine. Guidelines for the use of antifungal agents in patients with invasive fungal infections in Taiwan. J Microbiol Immunol Infect. 2006;39:523-5.

19. Infectious Diseases Society of Taiwan; Hematology Society of Taiwan; Taiwan Society of Pulmonary and Critical Care Medicine; Medical Foundation in Memory of Dr Deh-Lin Cheng; Foundation of Professor Wei-Chuan Hsieh for Infectious Diseases Research and Education; CY Lee's Research Foundation for Pediatric Infectious Diseases and Vaccines. Guidelines for the use of antifungal agents in patients with invasive fungal infections in Taiwan--revised 2009. J Microbiol Immunol Infect. 2010;43:258-63.

20. Berlow ME, Spirt BA, Weil L. CT follow-up of hepatic and splenic fungal microabscesses. J Comput Assist Tomogr. 1984;8:42-5.

21. Kauffman CA, Bradley SF, Ross SC, Weber DR. Hepatosplenic candidiasis: successful treatment with fluconazole. Am J Med. 1991;91:137-41.

22. Görg C, Weide R, Schwerk WB, Köppler H, Havemann K. Ultrasound evaluation of hepatic and splenic microabscesses in the immunocompromised patient: sonographic patterns, differential diagnosis, and follow-up. J Clin Ultrasound. 1994;22:525-9.
23. Shoham S, Levitz SM. The immune response to fungal infections. Br J Haematol. 2005:129:569-82.

24. van Prehn J, Menke-van der Houven van Oordt CW, de Rooij ML, Meijer E, Bomers MK, van Dijk K. Hepatosplenic Candidiasis Without Prior Documented Candidemia: An Underrecognized Diagnosis? Oncologist. 2017;22:989-94

25. Sun HY, Chiu YS, Tang JL, Wang JL, Chang SC, Chen YC. The usefulness of the Platelia Candida antigen in a patient with acute lymphocytic leukemia and chronic disseminated candidiasis. Med Mycol. 2006;44:647-50.

26. Marchetti O, Lamoth F, Mikulska M, Viscoli C, Verweij P, Bretagne S, et al. ECIL recommendations for the use of biological markers for the diagnosis of invasive fungal diseases in leukemic patients and hematopoietic SCT recipients. Bone Marrow Transplant. 2012:47:846-54.

27. Hot A, Maunoury C, Poiree S, Lanternier F, Viard JP, Loulergue P, et al. Diagnostic contribution of positron emission tomography with [18F] fluorodeoxyglucose for invasive fungal infections. Clin Microbiol Infect. 2011; 17:409-17.

28. Maertens J, Marchetti $O$, Herbrecht R, Cornely OA, Flückiger U, Frêre $P$, et al. European guidelines for antifungal management in leukemia and hematopoietic stem cell transplant recipients: summary of the ECIL 3--2009 update. Bone Marrow Transplant. 2011;46:709-18.

29. Tissot F, Agrawal S, Pagano L, Petrikkos G, Groll AH, Skiada A, et al. ECIL-6 guidelines for the treatment of invasive candidiasis, aspergillosis and mucormycosis in leukemia and hematopoietic stem cell transplant patients. Haematologica. 2017;102:433-44.

30. Bjerke JW, Meyers JD, Bowden RA. Hepatosplenic candidiasis--a contraindication to marrow transplantation? Blood. 1994;84:2811-4.

31. Antinori S, Milazzo L, Sollima S, Galli M, Corbellino M. Candidemia and invasive candidiasis in adults: a narrative review. Eur J Intern Med. 2016;34:21-8.

\section{Publisher's Note}

Springer Nature remains neutral with regard to jurisdictional claims in published maps and institutional affiliations.
Ready to submit your research? Choose BMC and benefit from:

- fast, convenient online submission

- thorough peer review by experienced researchers in your field

- rapid publication on acceptance

- support for research data, including large and complex data types

- gold Open Access which fosters wider collaboration and increased citations

- maximum visibility for your research: over $100 \mathrm{M}$ website views per year

At $\mathrm{BMC}$, research is always in progress.

Learn more biomedcentral.com/submissions 